\title{
Role of Immediate-Early Genes in Synaptic Plasticity and Neuronal Ensembles Underlying the Memory Trace
}

\author{
Keiichiro Minatohara, Mika Akiyoshi and Hiroyuki Okuno* \\ Medical Innovation Center/SK Project, Graduate School of Medicine, Kyoto University, Kyoto, Japan
}

In the brain, neuronal gene expression is dynamically changed in response to neuronal activity. In particular, the expression of immediate-early genes (IEGs) such as egr-1, c-fos, and Arc is rapidly and selectively upregulated in subsets of neurons in specific brain regions associated with learning and memory formation. IEG expression has therefore been widely used as a molecular marker for neuronal populations that undergo plastic changes underlying formation of long-term memory. In recent years, optogenetic and pharmacogenetic studies of neurons expressing c-fos or Arc have revealed that, during learning, IEG-positive neurons encode and store information that is required for memory recall, suggesting that they may be involved in formation of the memory trace. However, despite accumulating evidence for the role of IEGs in synaptic plasticity, the molecular and cellular mechanisms associated with this process remain unclear. In this review, we first summarize recent literature concerning the role of IEGexpressing neuronal ensembles in organizing the memory trace. We then focus on the physiological significance of IEGs, especially Arc, in synaptic plasticity, and describe our

OPEN ACCESS

Edited by: Peter K. Giese, King's College London, UK

Reviewed by: Naoki Matsuo, Osaka University, Japan Marco Peters, Dart NeuroScience LLC, USA

*Correspondence: Hiroyuki Okuno okuno@sk.med.kyoto-u.ac.jp

Received: 14 October 2015 Accepted: 03 December 2015 Published: 05 January 2016

Citation:

Minatohara K, Akiyoshi M and Okuno H (2016) Role of Immediate-Early Genes in Synaptic Plasticity and Neuronal Ensembles

Underlying the Memory Trace.

Front. Mol. Neurosci. 8:78. doi: 10.3389/fnmol.2015.00078 hypotheses about the importance of Arc expression in various types of input-specific circuit reorganization. Finally, we offer perspectives on Arc function that would unveil the role of IEG-expressing neurons in the formation of memory traces in the hippocampus and other brain areas.

Keywords: immediate-early gene, c-fos, Arc, synaptic plasticity, neuronal ensemble, memory trace

\section{INTRODUCTION}

Optogenetics and pharmacogenetics have become indispensable techniques to interrogate neuronal populations and circuits that underlie specific physiological functions and behavior (Deisseroth, 2015; Urban and Roth, 2015). In particular, combination of these techniques with cellular labeling/tagging specific to active neuronal ensembles has allowed elucidation of the physiological significance of neuronal ensembles in memory formation, storage, and recall. This review aims to provide an overview of recent understanding of memory traces in the brain and to help understand the molecular and cellular mechanisms underlying the memory trace by discussing two major topics: (I) immediate-early gene (IEG)-expressing neuronal ensembles as memory traces, and (II) the roles of Arc in synaptic plasticity and memory formation. Below, we begin with a brief background describing relationships between memory, synaptic plasticity, and IEGs.

Long-lasting forms of synaptic plasticity such as long-term potentiation (LTP) and long-term depression (LTD) are fundamental cellular mechanisms underlying learning and 
memory (Bliss and Collingridge, 2013). Induction of LTP occurs concomitantly with learning in the hippocampus of freely moving animals and is known to preclude subsequent electrical induction of LTP in the hippocampus (Whitlock et al., 2006). Conversely, prior massive induction of hippocampal LTP is also known to interfere with spatial memory formation (Barnes et al., 1994). A recent study has demonstrated that in vivo artificial induction of LTD impaired recall of associative memory, which was restored by subsequent LTP induction (Nabavi et al., 2014). Taken together, these findings suggest that a causal relationship exists between long-term synaptic plasticity and memory processes.

The molecular mechanisms underlying LTP have also been extensively investigated. Following plasticity-inducing synaptic input, $\mathrm{Ca}^{2+}$ entry through $N$-methyl-D-aspartate (NMDA)type receptors (NMDARs) plays a critical role in the onset of LTP via facilitation of $\alpha$-amino-3-hydroxy-5-methyl-4isoxazolepropionic (AMPA) receptor (AMPAR) recruitment to the potentiated post-synaptic sites (Collingridge et al., 1983; Kessels and Malinow, 2009). Furthermore, NMDAR-associated $\mathrm{Ca}^{2+}$ influx influences stabilization of LTP through activation of intracellular signaling cascades that subsequently promote mRNA and protein synthesis (Kandel et al., 2014). The blockade of these pathways using NMDAR antagonists (e.g., APV) or protein synthesis inhibitors (e.g., anisomycin) results in failure of the establishment of persistent LTP and impairment in formation of long-term memory (LTM; Gold, 2008; Redondo and Morris, 2011). Although these studies suggest that specific genes, induced during LTP, encode plasticity-related proteins (PRPs) required for LTP maintenance and memory formation, the identity of these genes remains unknown. A subset of plasticity-evoked, stimuli-induced genes, known as IEGs, has been implicated in the above events because of their rapid and transient responsiveness to synaptic activation (Okuno, 2011). For example, expression of IEGs such as egr-1 (zif268/krox-24), c-fos, and $\operatorname{Arc}$ ( $\arg 3.1)$, is rapidly upregulated after neuronal activation associated with pharmacologically induced convulsive and sensory stimuli (Morgan et al., 1987; Saffen et al., 1988; Link et al., 1995; Lyford et al., 1995). Behavioral tasks also induce IEG expression in neurons; such IEG-expressing neurons are distributed across a wide variety of brain regions (Rosen et al., 1998; Guzowski et al., 1999; Vann et al., 2000; Hall et al., 2001; Ramirez-Amaya et al., 2005). In the following sections, we describe studies analyzing the behavioral-induced IEG expression related to memory trace formation in more detail.

\section{IEG-EXPRESSING NEURONAL ENSEMBLES AS MEMORY TRACES}

\section{Induction of IEG Expression in Cell Ensembles Related to Cognitive Information Processing}

Immediate-early genes such as $A r c, c-f o s$, and egr-1 are induced in specific brain regions during neuronal activity associated with behavioral tasks. In the hippocampus, a center of declarative memory formation, rapid transcription of IEGs occurs during hippocampal-dependent learning paradigms including Morris water maze, novel environment exposure, and contextual fear conditioning (CFC; Guzowski et al., 1999, 2001; Vann et al., 2000; Hall et al., 2001; Ramirez-Amaya et al., 2005; Mamiya et al., 2009). Arc transcription is activated in a constant population (about $40 \%$ ) of CA1 neurons following exposure to a novel environment (Guzowski et al., 1999, 2006; Vazdarjanova et al., 2002). This proportion is similar to the percentage of activated neurons mapped using electrophysiology (Guzowski et al., 2006), suggestive of a strong correlation between neuronal activity and Arc expression. In addition to the hippocampus, other brain regions also contain IEG-positive neurons activated during learning and memory. Fear conditioning results in rapid IEG expression in the lateral amygdala (Rosen et al., 1998; Hall et al., 2001; Reijmers et al., 2007; Ploski et al., 2008), suggesting that these IEG-expressing neurons may be associated with emotional memory formation (Ploski et al., 2008; Maddox and Schafe, 2011).

The RNA transcripts of several IEGs, including Arc, egr-1 and homerla, first appear in the nucleus minutes after neuronal activation and are subsequently transferred to the cytoplasm (Guzowski et al., 1999). This temporally distinctive localization of IEG mRNA permits the differential labeling of activated neurons at different time points. This labeling method, known as cellular compartment analysis of temporal activity by fluorescent in situ hybridization (catFISH), has shown that sequential exposure to different environments induces IEG mRNA expression in distinct neuronal ensembles within the hippocampus, while sequential exposure to identical environments induces IEG mRNA in the same ensembles, indicating that activity-dependent IEG expression reflects spatial information processing in the hippocampus (Guzowski et al., 1999).

\section{Neuronal Ensembles with IEG Expression are Part of the Memory Trace}

Our understanding of the role of IEG-expressing neuronal ensembles in fear memory formation has been dramatically enhanced by recent studies using optogenetic and pharmacogenetic manipulation of neuronal activity in these ensembles. The CFC paradigm is designed to create an association between a neutral conditioned stimulus (e.g., chamber exposure) and an aversive unconditioned stimulus (e.g., foot shock; LeDoux, 1992). If an animal forms a fear memory through conditioning, freezing behavior is observed when the animal is re-exposed to the conditioned stimulus alone. Activated neurons during CFC transiently express IEGs (Hall et al., 2001; Mamiya et al., 2009). Moreover, expression of light-gated ion channels such as Channelrhodopsin 2 (ChR2) and Archaerhodopsin (Arch-T) or ligand-gated G-proteincoupled receptors such as designer receptors exclusively activated by designer drugs (DREADDs) in neurons under the control of IEG promoters permits manipulation of the activity of IEG-expressing neurons responding to specific training experiences (Figure 1; Neves et al., 2008). Figure 2 summarizes recent evidence concerning optogenetic and pharmacogenetic 


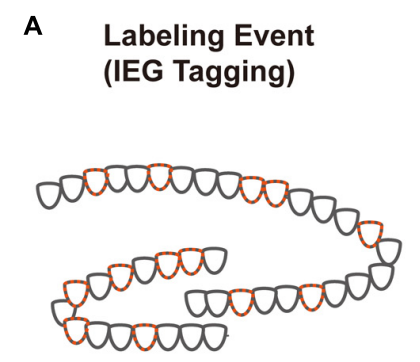

Activated Neuron

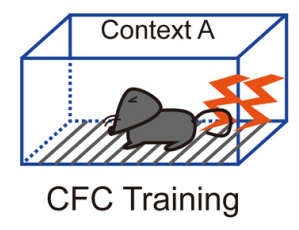

B
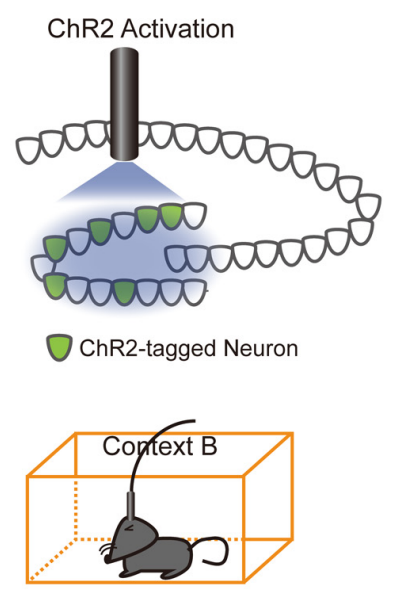

Freezing
C

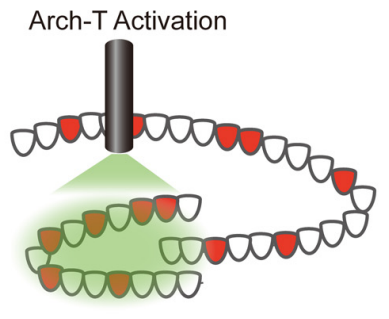

Arch-T-tagged Neuron

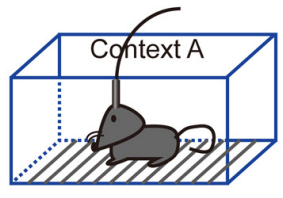

NOT Freezing

FIGURE 1 | Schematics of the optogenetic intervention experiments. (A) DG neurons activated during contextual fear conditioning (CFC) training in a conditioned context (context A) were labeled with either Channelrhodopsin 2 (ChR2; B) or Archaerhodopsin (Arch-T; C), whose expression is controlled by IEG promoters. (B) Trained mice show freezing responses during unconditioned context (context B) exposure only when the c-fos-positive ensemble expressing ChR2 was reactivated by blue light illumination (Liu et al., 2012). (C) Consistent with the ChR2 reactivation data, trained mice show no freezing responses to conditioned context A only when neuronal activity of the Arc-positive ensemble expressing Arch-T was inhibited by green light illumination (Denny et al., 2014).

\begin{tabular}{|c|c|c|c|c|c|c|}
\hline Driver & Effector & Labeling Event & $\begin{array}{l}\text { Target } \\
\text { Region }\end{array}$ & Manipulation Event & Main Result & Reference \\
\hline c-fos-tTA Tg & $\begin{array}{l}\text { AAV9-TRE-ChR2 } \\
\text { (Activation) }\end{array}$ & Ctx. A + US & DG & Ctx.B + Light & Freezing in Ctx. B during reactivation & $\begin{array}{l}\text { Liu et al., } \\
2012\end{array}$ \\
\hline c-fos-tTA Tg & $\begin{array}{l}\text { tetO-ChEF Tg } \\
\text { (Activation) }\end{array}$ & Ctx. A + US & RSC & $\begin{array}{c}\text { Ctx. B + Light } \\
+ \text { Hippocampal Inhibition }\end{array}$ & $\begin{array}{l}\text { Freezing in Ctx. B during reactivation } \\
\text { even in hippocampal activity inhibition }\end{array}$ & $\begin{array}{l}\text { Cowansage et al., } \\
2014\end{array}$ \\
\hline c-fos-tTA Tg & $\begin{array}{l}\text { tetO-hM3Dq Tg } \\
\text { (Activation) }\end{array}$ & Ctx. A & $\begin{array}{l}\text { Whole } \\
\text { Brain }\end{array}$ & Ctx. B + CNO + US & $\begin{array}{l}\text { Decrease in freezing in } \mathrm{Ctx} \text {. B without } \mathrm{CNO} \text {, } \\
\text { and recovered freezing with } \mathrm{CNO}\end{array}$ & $\begin{array}{l}\text { Garner et al. } \\
2012\end{array}$ \\
\hline c-fos-tTA Tg & $\begin{array}{l}\text { AAV9-TRE-ChR2 } \\
\text { (Activation) }\end{array}$ & Ctx.A & DG & Ctx. B + Light + US & Creating a false fear memory in Ctx. A & $\begin{array}{l}\text { Ramirez et al., } \\
2013\end{array}$ \\
\hline c-fos-tTA Tg & $\begin{array}{l}\text { AAV9-TRE-ChR2 } \\
\text { (Activation) }\end{array}$ & $\begin{array}{l}\text { Fear Conditioning or } \\
\text { Reward Conditioning }\end{array}$ & DG, BLA & $\begin{array}{l}\text { Reward Conditioning }+ \text { Light } \\
\text { or Fear Conditioning }+ \text { Light }\end{array}$ & $\begin{array}{l}\text { Conversion between fear and reward memory } \\
\text { after artificial DG reactivation }\end{array}$ & $\begin{array}{l}\text { Redondo et al., } \\
2014\end{array}$ \\
\hline c-fos-tTA Tg & $\begin{array}{l}\text { LV-TRE-ChR2 } \\
\text { (Activation) }\end{array}$ & $\begin{array}{l}\text { Ctx. A and } \\
\text { US in Ctx. B }\end{array}$ & $\begin{array}{l}\text { CA1, } \\
\text { BLA }\end{array}$ & $\begin{array}{l}\text { Synchronous Activation of } \\
\text { DG and BLA in Home Cage }\end{array}$ & $\begin{array}{l}\text { Increased freezing behavior in Ctx. B } \\
\text { after synchronous activation }\end{array}$ & $\begin{array}{l}\text { Ohkawa et al., } \\
2015\end{array}$ \\
\hline c-fos-tTA Tg & $\begin{array}{l}\text { AAV9-TRE-ChR2 } \\
\text { (Activation) }\end{array}$ & $\begin{aligned} & \text { Ctx. A + US } \\
+ & \text { Anisomycin }\end{aligned}$ & DG & Ctx. B + Light & $\begin{array}{l}\text { Freezing in Ctx. B during reactivation } \\
\text { even in amnesic mice }\end{array}$ & $\begin{array}{l}\text { Ryan et al., } \\
2015\end{array}$ \\
\hline $\begin{array}{l}\text { c-fos-tTA/ } \\
\text { tetO-Cre Tg }\end{array}$ & $\begin{array}{l}\text { AAV-FLEX-Arch-T } \\
\text { (Inactivation) }\end{array}$ & Ctx. A + US & CA1 & Ctx. A + Light & $\begin{array}{l}\text { Decrease in freezing in Ctx. A } \\
\text { during light-induced inactivation }\end{array}$ & $\begin{array}{l}\text { Tanaka et al., } \\
2014\end{array}$ \\
\hline c-fos-tTA Tg & $\begin{array}{l}\text { tetO-TeNT Tg } \\
\text { (Inhibition) }\end{array}$ & Ctx. A + US & $\begin{array}{l}\text { Whole } \\
\text { Brain }\end{array}$ & $\begin{array}{l}\text { Continuous Inhibition } \\
\text { from the Learning Event }\end{array}$ & Impaired fear memory even after relearning & $\begin{array}{l}\text { Matsuo, } \\
2015\end{array}$ \\
\hline $\begin{array}{c}\text { Arc- } \\
\text { CreERT2 Tg }\end{array}$ & $\begin{array}{l}\text { R26R-CAG-STOP- } \\
\text { floxed-Arch-T Tg } \\
\text { (Inactivation) }\end{array}$ & Ctx. A + US & CA3, DG & Ctx. A + Light & $\begin{array}{l}\text { Decrease in freezing in Ctx. A } \\
\text { during light-induced inactivation }\end{array}$ & $\begin{array}{l}\text { Denny et al., } \\
2014\end{array}$ \\
\hline
\end{tabular}

FIGURE 2 | A compendium of the current literature investigating the role of IEG-expressing neurons in memory formation. Driver transgenes promote the expression of the effector proteins that can manipulate neuronal activity. Labeling events induce effector protein expression via the driver transgenes. During activity manipulation events, IEG-expressing neurons are activated or inactivated by applying the trigger (e.g., light or clozapine- $N$-oxide; $C N O$ ). Tg, transgenic animal; AAV, adeno-associated virus; LV, lentivirus; tTA, tetracycline transactivator; tetO, tetracycline operator; TRE, tetracycline response element; ChEF, channelrhodopsin chimeric variant; Ctx, context; US, unconditioned stimulus (e.g., electrical foot shock); FLEX, flip-excision (double floxed system); TeNT, tetanus toxin.

manipulation of IEG-expressing neurons. The seminal study by Liu et al. (2012) that demonstrated involvement of IEGexpressing neurons in the memory trace used two transgene components, c-fos-tTA transgenic (tg) mice and TRE-ChR2 adeno-associated viral (AAV) vectors, by which ChR2 was expressed via a c-fos promoter only during an off-doxycycline (off-Dox) phase (Liu et al., 2012). These mice were subjected to CFC training in a conditioning chamber (context A) without 
Dox to label c-fos-positive ensembles with ChR2. After $24 \mathrm{~h}$, activation of ChR2-expressing c-fos ensembles using blue light illumination under a distinct neutral context (context B) elicited freezing responses only during illumination, suggesting that reactivation of $\mathrm{c}$-fos ensembles formed during CFC training was sufficient for the retrieval of the fear memory (Figure 1B). Inactivation of c-fos-positive ensembles in the dorsal CA1 region of the hippocampus expressing Arch-T has also been shown to impair memory recall (Tanaka et al., 2014). Similar to c-fos studies, optogenetic suppression of neuronal activity of Arc-positive neurons in hippocampal CA3 or dentate gyrus (DG), labeled with Arch-T during CFC training (context A), significantly impaired memory retrieval during re-exposure to the identical context (Figure 1C; Denny et al., 2014). Conversely, mice showed intact memory retrieval during suppression of Arc-positive ensembles responding to context B, supporting the concept of specificity in Arc-expressing ensembles (Denny et al., 2014). These studies indicate that reactivation of IEG ensembles represents a critical event underlying retrieval of fear memories.

Optogenetic or pharmacogenetic activation of c-fos-positive neurons related to the neutral context $\mathrm{B}$ during fear memory training in context A interfere with memory encoding by generating a hybrid contextual fear memory (Garner et al., 2012; Ramirez et al., 2013). Such a hybrid contextual fear memory creates a "false" memory for a neutral context (Ramirez et al., 2013). These studies demonstrated the physiological importance of IEG-expressing neuronal ensembles in memory encoding and suggested that IEG expression during CFC training may help integrate the activated ensembles into the neuronal circuits for fear memory formation. It should be noted that, whereas the pharmacogenetic study employed a systemic agonist injection to activate c-fos-positive ensembles throughout the whole brain (Garner et al., 2012), the optogenetic studies used focal optical fiber implantation to manipulate activities of IEG-positive neurons within the relevant subregion of hippocampus (i.e., DG, CA3, or CA1; Liu et al., 2012; Ramirez et al., 2013; Denny et al., 2014; Figure 2). Although these pharmacogenetic and optogenetic manipulations yielded overall consistent behavioral outputs of fear memory recall, it remains elusive whether brain-wide pharmacogenetic activation and hippocampal subregion-specific optogenetic manipulation deal with the same content of memory as natural memory retrieval. Especially because each sub-hippocampal region has been shown to have a distinct function in processing spatial information (e.g., pattern separation in DG and pattern completion in CA3; Nakazawa et al., 2002; McHugh et al., 2007), artificial activity manipulation of IEGexpressing ensembles in specific hippocampal subregions may result in different types of memory and information processing.

Several studies attempted to associate memory traces in the hippocampus and basolateral amygdala (BLA) by optogenetic neuronal activation. Simultaneous optogenetic co-activation of c-fos ensembles in the BLA (responding to a fear experience) with c-fos-expressing CA1 neurons (encoding a neutral context) generated a new fear memory associated with the neutral context (Ohkawa et al., 2015). Similarly, c-fos ensembles in the DG encoding a rewarding context can be changed to a fear memory trace via optical reactivation of the DG reward-related ensemble during fear conditioning (Redondo et al., 2014). These data suggest that IEG-expressing ensembles in brain regions such as the hippocampus and amygdala integrate to create associative fear memory. Consistent with this theory, a recent study demonstrated a critical role of the hippocampal-neocortical network formed by IEG-expressing neurons (Cowansage et al., 2014). The authors first showed that pharmacological blockade of hippocampal activity impaired fear memory retrieval, consistent with previous observations (Kitamura et al., 2009; Wiltgen et al., 2010). When c-fos-positive ensembles in the retrosplenial cortex (RSC) formed during training were optogenetically reactivated, the impairment of fear memory retrieval caused by hippocampal activity blockade was restored (Cowansage et al., 2014), suggesting that hippocampal neurons may contribute to fear memory retrieval by reactivating c-fos-positive memory ensembles distributed in the neocortical areas, including the RSC. Furthermore, suppression of hippocampal c-fos ensemble reactivation resulted in failure of the reactivation of $c$-fos memory ensembles in several cortical regions (Tanaka et al., 2014), indicating that IEG-expressing neurons distributed across distinct brain regions directly or indirectly activate each other and are crucial to forming and/or recalling a fear memory (Cowansage et al., 2014; Tanaka et al., 2014; Matsuo, 2015).

These hippocampal-cortical memory trace interactions at the early phase of memory formation and maintenance may stimulate reconsideration of a conventional view of system consolidation that assumes a slow, sequential involvement of cortical areas in memory trace formation (Frankland and Bontempi, 2005). Indeed, recent studies have shown hippocampal-dependent learning tasks immediately induce IEG expression in the cortical areas (Tse et al., 2011; Czajkowski et al., 2014). Furthermore, cortical activation in an early phase of memory formation induces functional changes in cortical neurons (Bero et al., 2014) and contributes to late-phase (remote) memory formation (Lesburgueres et al., 2011), suggesting that early phase (recent) memory trace exists in the neocortex as well as the hippocampus. The observations of Cowansage et al. (2014), in which the activation of c-fos ensembles in the RSC could evoke fear responses even in the absence of hippocampal activity, support the parallel encoding processing of fear memory in the neocortex and in the hippocampus shortly after learning. These results do not indicate, however, that hippocampal memory trace is unnecessary for natural retrieval processes, because "artificial" cortical activation could be sufficient to override the requirement of the hippocampus for contextual memory.

\section{c-fos Memory Trace Largely Overlaps with Other IEG-Expressing Ensembles}

The majority of studies concerning memory trace have focused on cell ensembles expressing c-fos (Figure 2). Do c-fosexpressing ensembles also express other IEGs? Surprisingly, little information is available regarding how and to what extent 
c-fos-expressing neuronal ensembles overlap with neuronal ensembles expressing other IEGs during memory encoding, although the individual expression patterns of each IEG have been well documented. Double in situ hybridization (ISH) analysis on the same section indicated most neurons in the cortical regions coexpressed IEGs including c-fos, $\operatorname{Arc}$, and Nr4a1 after monocular stimulation or sleep deprivation (Thompson et al., 2010; Nakagami et al., 2013). In the hippocampal CA1 and CA3, Arc-expressing neurons responding to a context exposure were more largely overlapped with Homer1a-expressing ensembles responding to the same context exposure than to a different context exposure (Vazdarjanova and Guzowski, 2004). Immunofluorescence analysis of fearconditioned brain sections revealed that most DG neurons coexpressed Arc and Egr-1 regardless of their differential expression time courses (Lonergan et al., 2010). These studies suggest that most neuronal ensembles encoding the fear memory may simultaneously express various IEGs, although the extent to which IEG-positive ensembles responding to behavioral tasks overlap with other IEGs-positive ensembles remains elusive.

\section{Functional Characteristics of IEG-Expressing Neurons}

Recent studies have investigated the characteristics of IEGexpressing neurons when compared to non-expressing neurons. In cortical regions, increased spontaneous firing rates have been observed in somatosensory neurons expressing c-fos or Arc (Yassin et al., 2010), and Arc-expressing neurons in the frontal cortex exhibit persistent firing after motor learning (Ren et al., 2014). Fear conditioning has been shown to increase surface expression of calcium-permeable AMPARs (i.e., GluA1 subunit-containing AMPA receptors) within c-fos-expressing cortical neurons (Descalzi et al., 2012). In the hippocampus, novel environment exposure alters dendritic spine morphology in Arc-expressing hippocampal neurons (Kitanishi et al., 2009), while fear conditioning induces selective spine elimination in CA1 neurons expressing c-fos (Sanders et al., 2012). In contrast to CA1 neurons, DG neurons expressing c-fos exhibit showed the increased spine density and enhanced synaptic transmission associated with protein synthesis after CFC training (Ryan et al., 2015). Although these studies demonstrated that experiencedependent IEG induction correlated with functional changes in activated neurons, it remains unclear whether IEG expression induces the functional changes required to generate memory traces.

\section{THE ROLES OF ArC IN SYNAPTIC PLASTICITY AND MEMORY FORMATION}

Although optogenetic and pharmacogenetic interventional approaches have suggested an important involvement of IEGexpressing neurons in memory formation, it remains unclear how IEG expression during learning participates in incorporation of IEG-expressing ensembles into the memory trace. In contrast to studies of memory traces encoded by c-fos-positive ensembles, little is known concerning the biological and physiological effects of c-fos on synaptic plasticity and neuronal circuit reorganization, in part because c-fos encodes a transcription factor composing the AP-1 complex, whose target genes in neurons have yet to be fully characterized. In contrast to c-fos, several IEGs including $B D N F$, narp, homerla, and Arc are known to encode synaptic or secretory proteins directly affecting synaptic properties (Chowdhury et al., 2006; Chang et al., 2010; Roloff et al., 2010; Lu et al., 2013). Elucidation of these proteins would shed light on molecular mechanisms underlying the incorporation of IEGexpressing ensembles into the memory trace. In the following sections, we review recent findings regarding the role of Arc in synaptic plasticity and memory formation.

\section{Long-Term Memory Formation Requires Arc Induction}

Arc expression is required for LTM consolidation, but not for learning or short-term memory formation (STM; Plath et al., 2006). Arc knockout (KO) mice exhibit impaired consolidation of spatial and fear memories (Plath et al., 2006; Peebles et al., 2010; Yamada et al., 2011). Transient inhibition of Arc expression following infusion of Arc antisense oligodeoxynucleotides (ODNs) into the hippocampus, lateral amygdala, or anterior cingulate cortex inhibits memory consolidation (Guzowski et al., 2000; Ploski et al., 2008; Holloway and McIntyre, 2011; Nakayama et al., 2015). This memory impairment occurs only when Arc antisense ODNs are infused immediately following memory acquisition, suggesting that induction of $A r c$ expression in response to training experience is necessary for LTM formation.

\section{Synaptic Localization of Arc}

One unique characteristic of $A r c$ is that its mRNA and protein can be targeted to dendritic compartments of neurons. Within minutes of neuronal activation triggered by behavioral events, Arc mRNA is expressed in the nucleus and subsequently transported through the cytoplasm into the dendrites (Wallace et al., 1998; Guzowski et al., 1999). Localization of dendritic mRNA is also regulated by synaptic activity; both Arc mRNA and protein accumulate in activated dendrites receiving high frequency stimulus (HFS; Steward et al., 1998; Moga et al., 2004). Biochemical and electron microscopic studies have confirmed the post-synaptic localization of Arc protein (Moga et al., 2004; Rodriguez et al., 2005; Chowdhury et al., 2006). Since Arc protein does not have obvious catalytic or other known functional domains, it is believed to function by interacting with other post-synaptic proteins. Arc interacts with endophilin and dynamin to form an endocytic complex believed to be involved in post-synaptic AMPAR trafficking, since Arc overexpression or knockout decreases or increases surface AMPAR expression, respectively (Chowdhury et al., 2006). Moreover, the effect of Arc on AMPAR regulation disappears when the interaction of Arc with endophilin/dynamin is disrupted (Chowdhury et al., 2006; Rial Verde et al., 2006). Although Arc facilitates AMPAR endocytosis, it does not appear 
to directly interact with AMPAR. A recent study revealed that the interaction of Arc with TARP $\gamma 2$ (stargazin) is required for Arc-dependent AMPAR synaptic scaling (see below; Zhang et al., 2015).

\section{Roles of Arc in Synaptic Scaling and Synaptic Plasticity}

Neurons possess the ability to maintain their excitability within a certain dynamic range by modifying surface AMPAR expression on synapses in response to changes in synaptic input, without affecting the relative balance between strong and weak synapses. These cellular changes have been termed "homeostatic" plasticity (Turrigiano, 2008), and induction of $A r c$ by neuronal activation and synaptic AMPAR endocytosis provide this IEG with a function in this process. Arc KO neurons in culture lack homeostatic AMPAR scaling (Shepherd et al., 2006) and Arc KO mice exhibit a deficit of synaptic scaling in response to sensory deprivation (McCurry et al., 2010). The expression of Arc appears to be required for cellular synaptic scaling in response to neuronal activity.

Arc-dependent AMPAR endocytosis is also involved in induction of LTD (Plath et al., 2006; Park et al., 2008). Activation of metabotropic glutamate receptors (mGluRs) rapidly induces $A r c$ translation, which is necessary for expression of mGluR-dependent LTD (Park et al., 2008) and suggests that $\operatorname{Arc}$ protein plays an important role in both input-specific synaptic plasticity and cell-wide synaptic scaling. Synaptic AMPAR downregulation by Arc appears irreconcilable with increases in Arc expression reported to occur following LTP-inducing stimulus and the transport of Arc mRNA and protein into activated dendrites. However, recent observations that $A r c$ protein is preferentially transported to inactive dendritic spines by binding with the inactive form of CaMKII $\beta$ and that AMPAR is selectively decreased in inactive spines in which $A r c$ is accumulated (Okuno et al., 2012), may help explain this apparent incongruity. This Arc-dependent downregulation of AMPAR in inactive synapses, termed "inverse synaptic tagging," likely functions to increase the contrast of synaptic strength between active and inactive synapses following synaptic potentiation (Figure 3A). Taken together, consolidation of synaptic plastic changes responding to neuronal activity is achieved, in part, via regulation of the expression and localization of Arc protein, which is in turn involved in surface AMPAR endocytosis.

\section{Metaplastic Changes of Arc-Expressing Neurons}

While it has been well documented that Arc expression is robustly induced throughout the rodent brain following exposure to a novel environment, the significance and consequence of its upregulation remain unclear. Several studies have revealed that Arc induction is associated with alteration of neuronal network properties, thereby facilitating consolidation of otherwise labile memories. For example, Arc-expressing neurons responding to novel environment exposure are preferentially reactivated during subsequent spontaneous hippocampal ripples, which are crucial for memory consolidation (Mizunuma et al., 2014). Arc may also be involved in a specific type of memory facilitating process called "behavioral tagging" (Moncada and Viola, 2007; Ballarini et al., 2009; Wang et al., 2010; Moncada et al., 2011). During "behavioral tagging," weak training tasks inducing STMs that last for only a few hours can produce LTM that lasts for over $24 \mathrm{~h}$ when accompanied by a novel experience that stimulates protein synthesis. This experience-dependent LTM facilitation is assumed to rely on PRPs synthesized during the novel experience, which would serve to induce and maintain plastic changes in the synapses "tagged" during the weak training. The suppression of Arc induction using ODNs during a novel experience inhibited LTM formation of weak behavioral tasks following the novel experience (Martinez et al., 2012), indicating that Arc may function as a PRP to facilitate LTM formation. Together with the inverse tagging mechanism described above, Arc protein synthesized prior to weak training may enhance synaptic strength between potentiated and non-potentiated synapses during the training (Figure 3B). These findings suggest that Arc expression prior to a behavioral task may influence the priming of synaptic plasticity. The history of neuronal activation can facilitate LTP or LTD induction, e.g., antecedent neuronal activities can alter the threshold of LTP and/or LTD expression induced by subsequent inputs. This type of plastic change is called "metaplasticity" (Abraham, 2008), and Arc has been suggested to be a modulating factor in this process (Shepherd and Bear, 2011). To this end, Arc induction has been reported to facilitate LTD expression (Jakkamsetti et al., 2013). Arc-expressing hippocampal neurons responding to exposure to a novel environment showed unaltered excitatory synaptic responses compared with the surrounding non-expressing neurons, but also showed prime mGluR-dependent LTD. Exposure to a novel environment also promoted expression and transportation of Arc mRNA to activated dendrites, while subsequent mGluR stimulation induced the translation of $\operatorname{Arc}$ in stimulated synapses and facilitated AMPAR endocytosis to express LTD in primed neurons (Jakkamsetti et al., 2013; Figure 3C). Furthermore, repeated experience caused a decrease in synaptic inputs to activated neurons via the Arc LTD-priming mechanism (Jakkamsetti et al., 2013). Taken together, LTD priming by Arc contributes to synaptic reorganization, which may help establish spatial recognition. Behavioral tagging and LTD priming by Arc are not mutually exclusive and can occur in the same neurons simultaneously. Arc induction may also increase the flexibility of synaptic changes in responding to various forms of subsequent stimuli. For example, synapses activated during repeated exposure to the same environment will be weakened by priming and induction of LTD (Jakkamsetti et al., 2013; Figure 3D, arrow). Conversely, synaptic tagging and inverse tagging processes will heighten the contrast of synaptic strength in response to weak synaptic inputs (Figure 3D, arrow head). These synaptic modifications of Arc-expressing neurons will reorganize the activated circuits underlying memory trace formation (Figure 3D, right). 


\section{A Inverse Synaptic Tagging}
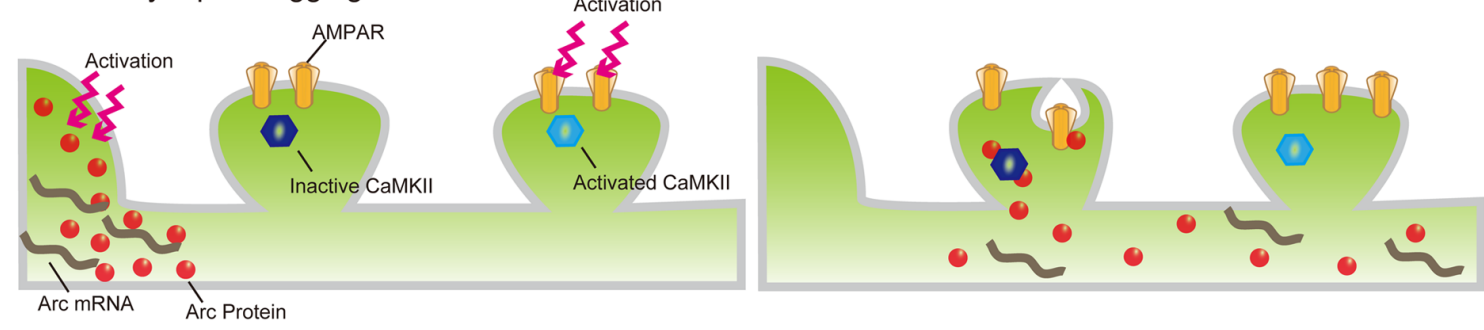

B Behavioral Tagging
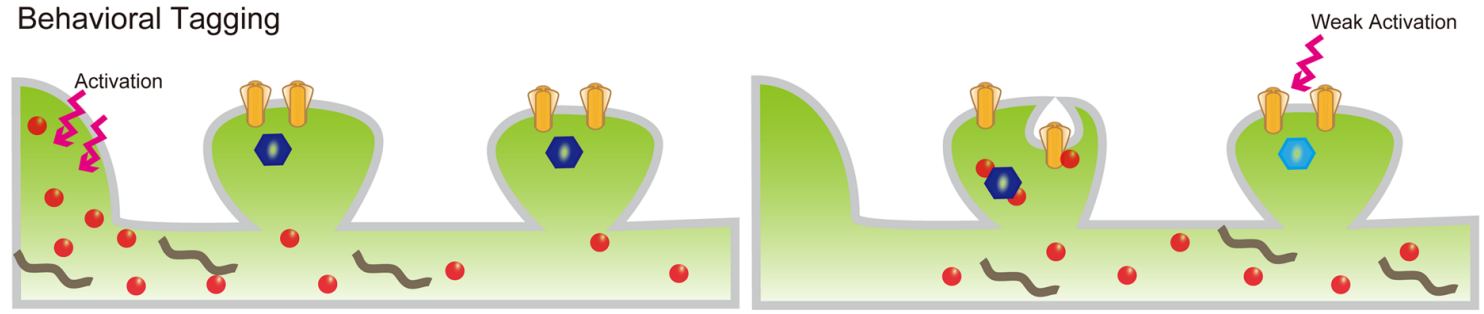

c LTD Priming
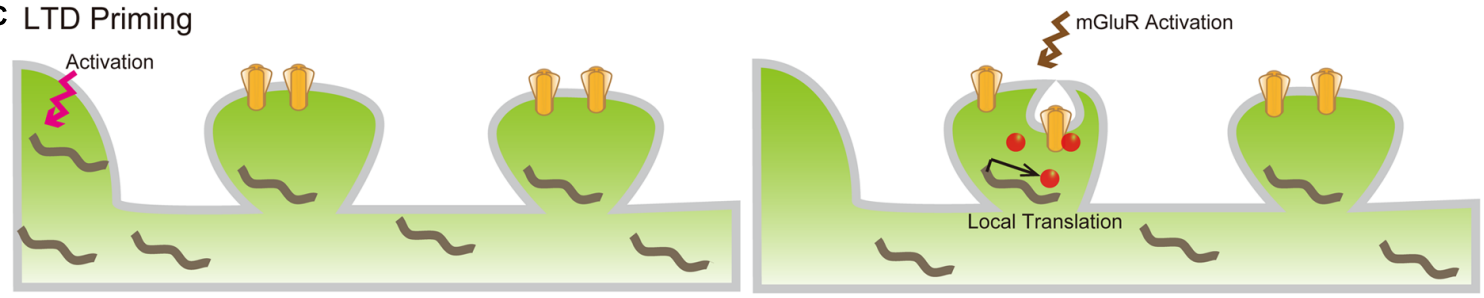

D

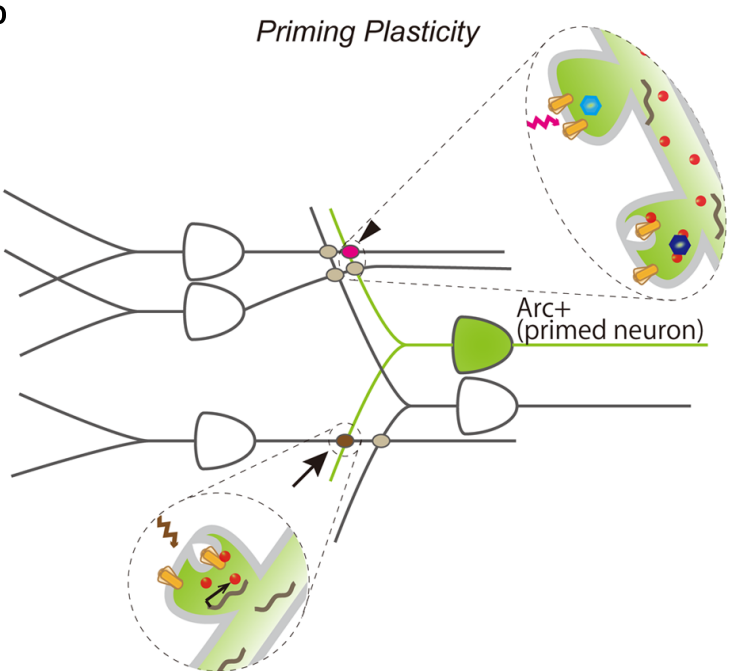

Circuit Reorganization

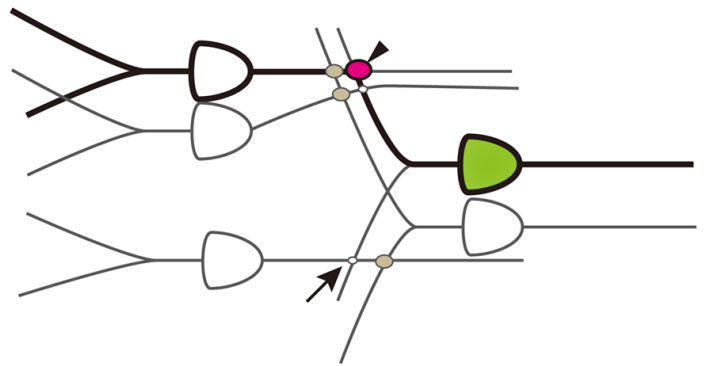

FIGURE 3 | Models of Arc mRNA and protein dynamics in inverse tagging (A), behavioral tagging (B), and LTD priming (C) processes. (A) Arc protein is synthesized in the soma following neuronal activation (left) and transported to an activated dendrite (right). Arc preferentially binds to the inactive form of CaMKIl and promotes AMPAR endocytosis in the inactive synapse, resulting in the increasing synaptic strength of the activated synapse. (B) A novel exploration task induces Arc protein expression (left). Arc protein diffuses throughout the dendrite, decreases AMPAR in the non-tagged synapse, and serves to maintain the enhanced synaptic strength of synapses transiently potentiated by a weak training (right). (C) Other types of behavioral experiences promote Arc mRNA synthesis in the nucleus and transport to nearby synaptic sites, but its translation is suspended (left). Subsequent mGluR activation promotes Arc translation from dendritically localized Arc mRNA, resulting in AMPAR internalization and LTD of the mGluR-activated synapse. (D) Arc expression plays a permissive role in inducing synapse-specific plastic changes to organize a new memory trace following a new behavior/experience. Arrow and arrowhead indicate LTD-primed and behavioral-tagged synapses, respectively. 


\section{CONCLUSION AND FUTURE DIRECTIONS}

In this review, we characterized the role of IEG-expressing neurons in memory formation and storage. Neurons activated during cognitive tasks induce IEGs and organize the memory trace (Figures 1 and 2). During memory formation and recall, hippocampal IEG-positive cells are co-activated alongside IEG-positive neurons in other brain areas, including the amygdala and neocortex, suggesting that IEG-positive neurons are preferentially connected with each other across brain regions. Furthermore, IEG-positive neurons exhibit functional synaptic changes that may underlie memory formation. We also summarized evidence for the role of Arc in synaptic plasticity and memory formation. Taken together, the literature suggests that continued characterization of the functional changes in Arcexpressing neurons will elucidate novel molecular mechanisms underlying memory formation and/or storage.

The expression of IEGs is dynamically regulated in response to neuronal activity in the brain; while many neurons only express IEG at basal levels, some neurons display rapid induction of IEG expression surpassing the basal level after learning (Rosen et al., 1998; Guzowski et al., 1999; Vann et al., 2000; Hall et al., 2001; Ramirez-Amaya et al., 2005). Such dual components of IEG expression raise an important but unsolved question: what is the significance of learning-induced, but not basal, IEG expression? Our current knowledge about IEG functions in memory largely depends on loss-of-function studies, most of which use conventional IEG KO animals. Such approach could demonstrate the necessity of IEGs, but not specifically learninginduced IEGs, in learning and memory. To address this issue, one possible strategy is to specifically suppress learning-induced IEGs by directly manipulating activity-dependent expression. The promoters and enhancers that contribute to activity-dependent expression of c-fos, $A r c$, and $B d n f$ have been characterized (Robertson et al., 1995; West et al., 2001; Kawashima et al., 2009). In fact, such a strategy has successfully dissected the role of activity-dependent expression of the Bdnf gene (Hong et al., 2008; Sakata et al., 2009, 2013). Bdnf possesses multiple promoters including promoter IV, which is responsible for activity-dependent $B d n f$ expression. When promoter IV is mutated, activity-dependent, but not basal, $B d n f$ expression is

\section{REFERENCES}

Abraham, W. C. (2008). Metaplasticity: tuning synapses and networks for plasticity. Nat. Rev. Neurosci. 9:387. doi: 10.1038/nrn2356

Ballarini, F., Moncada, D., Martinez, M. C., Alen, N., and Viola, H. (2009). Behavioral tagging is a general mechanism of long-term memory formation. Proc. Natl. Acad. Sci. U.S.A. 106, 14599-14604. doi: 10.1073/pnas.0907 078106

Barnes, C. A., Jung, M. W., McNaughton, B. L., Korol, D. L., Andreasson, K., and Worley, P. F. (1994). LTP saturation and spatial learning disruption: effects of task variables and saturation levels. J. Neurosci. 14, 5793-5806.

Barth, A. L., Gerkin, R. C., and Dean, K. L. (2004). Alteration of neuronal firing properties after in vivo experience in a FosGFP transgenic mouse. J. Neurosci. 24, 6466-6475. doi: 10.1523/JNEUROSCI.4737-03. 2004 greatly reduced and cortical circuit organization is altered (Hong et al., 2008; Sakata et al., 2009). Furthermore, hippocampal synaptic plasticity and behavioral flexibility are impaired (Sakata et al., 2013). Manipulations in the transcriptional cis-elements would therefore help elucidate a causal relationship between behaviorally related IEG induction and memory trace formation.

Recently, visualization of dynamic changes in IEG expression in vivo using fluorescent reporters under the control of the IEG promoter has been achieved (Barth et al., 2004; Wang et al., 2006; Eguchi and Yamaguchi, 2009; Grinevich et al., 2009; Kawashima et al., 2009). These techniques enable us to analyze neurons expressing IEGs while responding to behavioral tasks, facilitating the understanding of the physiological roles of activity-dependent IEG expression in synaptic plasticity and memory formation.

Furthermore, it is widely believed that dysregulation of neuronal activity and synaptic functions cause various types of cognitive disorders including autism, schizophrenia, and dementia, as these diseases are related to mutations in genes associated with activity-dependent gene expression and synaptic maturation (West and Greenberg, 2011; Purcell et al., 2014). Future studies analyzing the roles of activity- and/or behavioraldependent expression of Arc and other IEGs in synaptic plasticity may enhance our understanding of the pathogenesis and treatment of specific psychiatric and neurological disorders.

\section{AUTHOR CONTRIBUTIONS}

$\mathrm{KM}$, conceived the content and wrote the manuscript; MA, provided the ideas and discussions; HO, conceived the content, wrote the manuscript, and supervised the work.

\section{ACKNOWLEDGMENTS}

We apologize to the many authors we could not mention due to space limitations. We thank the members of SK project and Okuno lab for their support. This work was supported in part by research funds from Shionogi \& CO., LTD; by Grants-in-Aid for Scientific Research (KAKENHI 15H04258) from the Ministry of Education, Culture, Sports, Science and Technology of Japan; and by a research grant from Brain Science Foundation.

Bero, A. W., Meng, J., Cho, S., Shen, A. H., Canter, R. G., Ericsson, M., et al. (2014). Early remodeling of the neocortex upon episodic memory encoding. Proc. Natl. Acad. Sci. U.S.A. 111, 11852-11857. doi: 10.1073/pnas.1408 378111

Bliss, T. V., and Collingridge, G. L. (2013). Expression of NMDA receptordependent LTP in the hippocampus: bridging the divide. Mol. Brain 6:5. doi: 10.1186/1756-6606-6-5

Chang, M. C., Park, J. M., Pelkey, K. A., Grabenstatter, H. L., Xu, D., Linden, D. J., et al. (2010). Narp regulates homeostatic scaling of excitatory synapses on parvalbumin-expressing interneurons. Nat. Neurosci. 13, 1090-1097. doi: 10.1038/nn.2621

Chowdhury, S., Shepherd, J. D., Okuno, H., Lyford, G., Petralia, R. S., Plath, N., et al. (2006). Arc/Arg3.1 interacts with the endocytic machinery to regulate AMPA receptor trafficking. Neuron 52, 445-459. doi: 10.1016/j.neuron.2006.08.033 
Collingridge, G. L., Kehl, S. J., and McLennan, H. (1983). Excitatory amino acids in synaptic transmission in the Schaffer collateral-commissural pathway of the rat hippocampus. J. Physiol. 334, 33-46. doi: 10.1113/jphysiol.1983.sp 014478

Cowansage, K. K., Shuman, T., Dillingham, B. C., Chang, A., Golshani, P., and Mayford, M. (2014). Direct reactivation of a coherent neocortical memory of context. Neuron 84, 432-441. doi: 10.1016/j.neuron.2014.09.022

Czajkowski, R., Jayaprakash, B., Wiltgen, B., Rogerson, T., Guzman-Karlsson, M. C., Barth, A. L., et al. (2014). Encoding and storage of spatial information in the retrosplenial cortex. Proc. Natl. Acad. Sci. U.S.A. 111, 8661-8666. doi: $10.1073 /$ pnas.1313222111

Deisseroth, K. (2015). Optogenetics: 10 years of microbial opsins in neuroscience. Nat. Neurosci. 18, 1213-1225. doi: 10.1038/nn.4091

Denny, C. A., Kheirbek, M. A., Alba, E. L., Tanaka, K. F., Brachman, R. A., Laughman, K. B., et al. (2014). Hippocampal memory traces are differentially modulated by experience, time, and adult neurogenesis. Neuron 83, 189-201. doi: 10.1016/j.neuron.2014.05.018

Descalzi, G., Li, X. Y., Chen, T., Mercaldo, V., Koga, K., and Zhuo, M. (2012). Rapid synaptic potentiation within the anterior cingulate cortex mediates trace fear learning. Mol. Brain 5:6. doi: 10.1186/1756-6606-5-6

Eguchi, M., and Yamaguchi, S. (2009). In vivo and in vitro visualization of gene expression dynamics over extensive areas of the brain. Neuroimage 44, 1274-1283. doi: 10.1016/j.neuroimage.2008.10.046

Frankland, P. W., and Bontempi, B. (2005). The organization of recent and remote memories. Nat. Rev. Neurosci. 6, 119-130. doi: 10.1038/nrn1607

Garner, A. R., Rowland, D. C., Hwang, S. Y., Baumgaertel, K., Roth, B. L., Kentros, C., et al. (2012). Generation of a synthetic memory trace. Science 335 , 1513-1516. doi: 10.1126/science. 1214985

Gold, P. E. (2008). Protein synthesis inhibition and memory: formation vs amnesia. Neurobiol. Learn. Mem. 89, 201-211. doi: 10.1016/j.nlm.2007.10.006

Grinevich, V., Kolleker, A., Eliava, M., Takada, N., Takuma, H., Fukazawa, Y., et al. (2009). Fluorescent Arc/Arg3.1 indicator mice: a versatile tool to study brain activity changes in vitro and in vivo. J Neurosci Methods 184, 25-36. doi: 10.1016/j.jneumeth.2009.07.015

Guzowski, J. F., Lyford, G. L., Stevenson, G. D., Houston, F. P., McGaugh, J. L., Worley, P. F., et al. (2000). Inhibition of activity-dependent arc protein expression in the rat hippocampus impairs the maintenance of long-term potentiation and the consolidation of long-term memory. J. Neurosci. 20, 3993-4001.

Guzowski, J. F., McNaughton, B. L., Barnes, C. A., and Worley, P. F. (1999). Environment-specific expression of the immediate-early gene Arc in hippocampal neuronal ensembles. Nat. Neurosci. 2, 1120-1124. doi: $10.1038 / 16046$

Guzowski, J. F., Miyashita, T., Chawla, M. K., Sanderson, J., Maes, L. I., Houston, F. P., et al. (2006). Recent behavioral history modifies coupling between cell activity and Arc gene transcription in hippocampal CA1 neurons. Proc. Natl. Acad. Sci. U.S.A. 103, 1077-1082. doi: 10.1073/pnas.05055 19103

Guzowski, J. F., Setlow, B., Wagner, E. K., and McGaugh, J. L. (2001). Experiencedependent gene expression in the rat hippocampus after spatial learning: a comparison of the immediate-early genes Arc, c-fos, and zif268. J. Neurosci. 21, 5089-5098.

Hall, J., Thomas, K. L., and Everitt, B. J. (2001). Cellular imaging of zif268 expression in the hippocampus and amygdala during contextual and cued fear memory retrieval: selective activation of hippocampal CA1 neurons during the recall of contextual memories. J. Neurosci. 21, 2186-2193.

Holloway, C. M., and McIntyre, C. K. (2011). Post-training disruption of Arc protein expression in the anterior cingulate cortex impairs long-term memory for inhibitory avoidance training. Neurobiol. Learn. Mem. 95, 425-432. doi: 10.1016/j.nlm.2011.02.002

Hong, E. J., McCord, A. E., and Greenberg, M. E. (2008). A biological function for the neuronal activity-dependent component of Bdnf transcription in the development of cortical inhibition. Neuron 60, 610-624. doi: 10.1016/j.neuron.2008.09.024

Jakkamsetti, V., Tsai, N. P., Gross, C., Molinaro, G., Collins, K. A., Nicoletti, F., et al. (2013). Experience-induced Arc/Arg3.1 primes CA1 pyramidal neurons for metabotropic glutamate receptor-dependent long-term synaptic depression. Neuron 80, 72-79. doi: 10.1016/j.neuron.2013.07.020
Kandel, E. R., Dudai, Y., and Mayford, M. R. (2014). The molecular and systems biology of memory. Cell 157, 163-186. doi: 10.1016/j.cell.2014.03.001

Kawashima, T., Okuno, H., Nonaka, M., Adachi-Morishima, A., Kyo, N., Okamura, M., et al. (2009). Synaptic activity-responsive element in the Arc/Arg3.1 promoter essential for synapse-to-nucleus signaling in activated neurons. Proc. Natl. Acad. Sci. U.S.A. 106, 316-321. doi: 10.1073/pnas.0806518106

Kessels, H. W., and Malinow, R. (2009). Synaptic AMPA receptor plasticity and behavior. Neuron 61, 340-350. doi: 10.1016/j.neuron.2009.01.015

Kitamura, T., Saitoh, Y., Takashima, N., Murayama, A., Niibori, Y., Ageta, H., et al. (2009). Adult neurogenesis modulates the hippocampus-dependent period of associative fear memory. Cell 139, 814-827. doi: 10.1016/j.cell.2009. 10.020

Kitanishi, T., Ikegaya, Y., Matsuki, N., and Yamada, M. K. (2009). Experiencedependent, rapid structural changes in hippocampal pyramidal cell spines. Cereb. Cortex 19, 2572-2578. doi: 10.1093/cercor/bhp012

LeDoux, J. E. (1992). Brain mechanisms of emotion and emotional learning. Curr. Opin. Neurobiol. 2, 191-197. doi: 10.1016/0959-4388(92)90011-9

Lesburgueres, E., Gobbo, O. L., Alaux-Cantin, S., Hambucken, A., Trifilieff, P., and Bontempi, B. (2011). Early tagging of cortical networks is required for the formation of enduring associative memory. Science 331, 924-928. doi: $10.1126 /$ science. 1196164

Link, W., Konietzko, U., Kauselmann, G., Krug, M., Schwanke, B., Frey, U., et al. (1995). Somatodendritic expression of an immediate early gene is regulated by synaptic activity. Proc. Natl. Acad. Sci. U.S.A. 92, 5734-5738. doi: 10.1073/pnas.92.12.5734

Liu, X., Ramirez, S., Pang, P. T., Puryear, C. B., Govindarajan, A., Deisseroth, K., et al. (2012). Optogenetic stimulation of a hippocampal engram activates fear memory recall. Nature 484, 381-385. doi: 10.1038/nature 11028

Lonergan, M. E., Gafford, G. M., Jarome, T. J., and Helmstetter, F. J. (2010). Timedependent expression of Arc and zif268 after acquisition of fear conditioning. Neural Plast. 2010:139891. doi: 10.1155/2010/139891

Lu, B., Nagappan, G., Guan, X., Nathan, P. J., and Wren, P. (2013). BDNF-based synaptic repair as a disease-modifying strategy for neurodegenerative diseases. Nat. Rev. Neurosci. 14, 401-416. doi: 10.1038/nrn3505

Lyford, G. L., Yamagata, K., Kaufmann, W. E., Barnes, C. A., Sanders, L. K., Copeland, N. G., et al. (1995). Arc, a growth factor and activity-regulated gene, encodes a novel cytoskeleton-associated protein that is enriched in neuronal dendrites. Neuron 14, 433-445. doi: 10.1016/0896-6273(95)90299-6

Maddox, S. A., and Schafe, G. E. (2011). The activity-regulated cytoskeletalassociated protein (Arc/Arg3.1) is required for reconsolidation of a Pavlovian fear memory. J. Neurosci. 31, 7073-7082. doi: 10.1523/JNEUROSCI.112011.2011

Mamiya, N., Fukushima, H., Suzuki, A., Matsuyama, Z., Homma, S., Frankland, P. W., et al. (2009). Brain region-specific gene expression activation required for reconsolidation and extinction of contextual fear memory. J. Neurosci. 29, 402-413. doi: 10.1523/JNEUROSCI.4639-08.2009

Martinez, M. C., Alen, N., Ballarini, F., Moncada, D., and Viola, H. (2012). Memory traces compete under regimes of limited Arc protein synthesis: implications for memory interference. Neurobiol. Learn. Mem. 98, 165-173. doi: 10.1016/j.nlm.2012.05.007

Matsuo, N. (2015). Irreplaceability of neuronal ensembles after memory allocation. Cell Rep. 11, 351-357. doi: 10.1016/j.celrep.2015.03.042

McCurry, C. L., Shepherd, J. D., Tropea, D., Wang, K. H., Bear, M. F., and Sur, M. (2010). Loss of Arc renders the visual cortex impervious to the effects of sensory experience or deprivation. Nat. Neurosci. 13, 450-457. doi: 10.1038/nn. 2508

McHugh, T. J., Jones, M. W., Quinn, J. J., Balthasar, N., Coppari, R., Elmquist, J. K., et al. (2007). Dentate gyrus NMDA receptors mediate rapid pattern separation in the hippocampal network. Science 317, 94-99. doi: 10.1126/science.11 40263

Mizunuma, M., Norimoto, H., Tao, K., Egawa, T., Hanaoka, K., Sakaguchi, T., et al. (2014). Unbalanced excitability underlies offline reactivation of behaviorally activated neurons. Nat. Neurosci. 17, 503-505. doi: 10.1038/nn.3674

Moga, D. E., Calhoun, M. E., Chowdhury, A., Worley, P., Morrison, J. H., and Shapiro, M. L. (2004). Activity-regulated cytoskeletal-associated protein is localized to recently activated excitatory synapses. Neuroscience 125, 7-11. doi: 10.1016/j.neuroscience.2004.02.004 
Moncada, D., Ballarini, F., Martinez, M. C., Frey, J. U., and Viola, H. (2011). Identification of transmitter systems and learning tag molecules involved in behavioral tagging during memory formation. Proc. Natl. Acad. Sci. U.S.A. 108, 12931-12936. doi: 10.1073/pnas.1104495108

Moncada, D., and Viola, H. (2007). Induction of long-term memory by exposure to novelty requires protein synthesis: evidence for a behavioral tagging. J. Neurosci. 27, 7476-7481. doi: 10.1523/JNEUROSCI.1083-07.2007

Morgan, J. I., Cohen, D. R., Hempstead, J. L., and Curran, T. (1987). Mapping patterns of c-fos expression in the central nervous system after seizure. Science 237, 192-197. doi: 10.1126/science.3037702

Nabavi, S., Fox, R., Proulx, C. D., Lin, J. Y., Tsien, R. Y., and Malinow, R. (2014). Engineering a memory with LTD and LTP. Nature 511, 348-352. doi: 10.1038 /nature 13294

Nakagami, Y., Watakabe, A., and Yamamori, T. (2013). Monocular inhibition reveals temporal and spatial changes in gene expression in the primary visual cortex of marmoset. Front. Neural Circ. 7:43. doi: 10.3389/fncir.2013.00043

Nakayama, D., Iwata, H., Teshirogi, C., Ikegaya, Y., Matsuki, N., and Nomura, H. (2015). Long-delayed expression of the immediate early gene Arc/Arg3.1 refines neuronal circuits to perpetuate fear memory. J. Neurosci. 35, 819-830. doi: 10.1523/JNEUROSCI.2525-14.2015

Nakazawa, K., Quirk, M. C., Chitwood, R. A., Watanabe, M., Yeckel, M. F., Sun, L. D., et al. (2002). Requirement for hippocampal CA3 NMDA receptors in associative memory recall. Science 297, 211-218. doi: 10.1126/science.1071795

Neves, G., Cooke, S. F., and Bliss, T. V. (2008). Synaptic plasticity, memory and the hippocampus: a neural network approach to causality. Nat. Rev. Neurosci. 9, 65-75. doi: 10.1038/nrn2303

Ohkawa, N., Saitoh, Y., Suzuki, A., Tsujimura, S., Murayama, E., Kosugi, S., et al. (2015). Artificial association of pre-stored information to generate a qualitatively new memory. Cell Rep. 11, 261-269. doi: 10.1016/j.celrep.2015.03.017

Okuno, H. (2011). Regulation and function of immediate-early genes in the brain: beyond neuronal activity markers. Neurosci. Res. 69, 175-186. doi: 10.1016/j.neures.2010.12.007

Okuno, H., Akashi, K., Ishii, Y., Yagishita-Kyo, N., Suzuki, K., Nonaka, M., et al. (2012). Inverse synaptic tagging of inactive synapses via dynamic interaction of Arc/Arg3.1 with CaMKIIbeta. Cell 149, 886-898. doi: 10.1016/j.cell.2012. 02.062

Park, S., Park, J. M., Kim, S., Kim, J. A., Shepherd, J. D., Smith-Hicks, C. L., et al. (2008). Elongation factor 2 and fragile $\mathrm{X}$ mental retardation protein control the dynamic translation of Arc/Arg3.1 essential for mGluR-LTD. Neuron 59, 70-83. doi: 10.1016/j.neuron.2008.05.023

Peebles, C. L., Yoo, J., Thwin, M. T., Palop, J. J., Noebels, J. L., and Finkbeiner, S. (2010). Arc regulates spine morphology and maintains network stability in vivo. Proc. Natl. Acad. Sci. U.S.A. 107, 18173-18178. doi: 10.1073/pnas.1006546107

Plath, N., Ohana, O., Dammermann, B., Errington, M. L., Schmitz, D., Gross, C., et al. (2006). Arc/Arg3.1 is essential for the consolidation of synaptic plasticity and memories. Neuron 52, 437-444. doi: 10.1016/j.neuron.2006.08.024

Ploski, J. E., Pierre, V. J., Smucny, J., Park, K., Monsey, M. S., Overeem, K. A., et al. (2008). The activity-regulated cytoskeletal-associated protein (Arc/Arg3.1) is required for memory consolidation of pavlovian fear conditioning in the lateral amygdala. J. Neurosci. 28, 12383-12395. doi: 10.1523/JNEUROSCI.166208.2008

Purcell, S. M., Moran, J. L., Fromer, M., Ruderfer, D., Solovieff, N., Roussos, P., et al. (2014). A polygenic burden of rare disruptive mutations in schizophrenia. Nature 506, 185-190. doi: 10.1038/nature12975

Ramirez, S., Liu, X., Lin, P. A., Suh, J., Pignatelli, M., Redondo, R. L., et al. (2013). Creating a false memory in the hippocampus. Science 341, 387-391. doi: $10.1126 /$ science. 1239073

Ramirez-Amaya, V., Vazdarjanova, A., Mikhael, D., Rosi, S., Worley, P. F., and Barnes, C. A. (2005). Spatial exploration-induced Arc mRNA and protein expression: evidence for selective, network-specific reactivation. J. Neurosci. 25, 1761-1768. doi: 10.1523/JNEUROSCI.4342-04.2005

Redondo, R. L., Kim, J., Arons, A. L., Ramirez, S., Liu, X., and Tonegawa, S. (2014). Bidirectional switch of the valence associated with a hippocampal contextual memory engram. Nature 513, 426-430. doi: 10.1038/nature13725

Redondo, R. L., and Morris, R. G. (2011). Making memories last: the synaptic tagging and capture hypothesis. Nat. Rev. Neurosci. 12, 17-30. doi: $10.1038 /$ nrn 2963
Reijmers, L. G., Perkins, B. L., Matsuo, N., and Mayford, M. (2007). Localization of a stable neural correlate of associative memory. Science 317, 1230-1233. doi: 10.1126/science.1143839

Ren, M., Cao, V., Ye, Y., Manji, H. K., and Wang, K. H. (2014). Arc regulates experience-dependent persistent firing patterns in frontal cortex. J. Neurosci. 34, 6583-6595. doi: 10.1523/JNEUROSCI.0167-14.2014

Rial Verde, E. M., Lee-Osbourne, J., Worley, P. F., Malinow, R., and Cline, H. T. (2006). Increased expression of the immediate-early gene arc/arg3.1 reduces AMPA receptor-mediated synaptic transmission. Neuron 52, 461-474. doi: 10.1016/j.neuron.2006.09.031

Robertson, L. M., Kerppola, T. K., Vendrell, M., Luk, D., Smeyne, R. J., Bocchiaro, C., et al. (1995). Regulation of c-fos expression in transgenic mice requires multiple interdependent transcription control elements. Neuron 14, 241-252. doi: 10.1016/0896-6273(95)90282-1

Rodriguez, J. J., Davies, H. A., Silva, A. T., De Souza, I. E., Peddie, C. J., Colyer, F. M., et al. (2005). Long-term potentiation in the rat dentate gyrus is associated with enhanced Arc/Arg3.1 protein expression in spines, dendrites and glia. Eur. J. Neurosci. 21, 2384-2396. doi: 10.1111/j.1460-9568.2005.04068.x

Roloff, A. M., Anderson, G. R., Martemyanov, K. A., and Thayer, S. A. (2010). Homer 1a gates the induction mechanism for endocannabinoid-mediated synaptic plasticity. J. Neurosci. 30, 3072-3081. doi: 10.1523/JNEUROSCI.460309.2010

Rosen, J. B., Fanselow, M. S., Young, S. L., Sitcoske, M., and Maren, S. (1998). Immediate-early gene expression in the amygdala following footshock stress and contextual fear conditioning. Brain Res. 796, 132-142. doi: 10.1016/S00068993(98)00294-7

Ryan, T. J., Roy, D. S., Pignatelli, M., Arons, A., and Tonegawa, S. (2015). Memory. Engram cells retain memory under retrograde amnesia. Science 348, 1007-1013. doi: $10.1126 /$ science.aaa5542

Saffen, D. W., Cole, A. J., Worley, P. F., Christy, B. A., Ryder, K., and Baraban, J. M. (1988). Convulsant-induced increase in transcription factor messenger RNAs in rat brain. Proc. Natl. Acad. Sci. U.S.A. 85, 7795-7799. doi: 10.1073/pnas.85.20.7795

Sakata, K., Martinowich, K., Woo, N. H., Schloesser, R. J., Jimenez, D. V., Ji, Y., et al. (2013). Role of activity-dependent BDNF expression in hippocampal-prefrontal cortical regulation of behavioral perseverance. Proc. Natl. Acad. Sci. U.S.A. 110, 15103-15108. doi: 10.1073/pnas.1222872110

Sakata, K., Woo, N. H., Martinowich, K., Greene, J. S., Schloesser, R. J., Shen, L., et al. (2009). Critical role of promoter IV-driven BDNF transcription in GABAergic transmission and synaptic plasticity in the prefrontal cortex. Proc. Natl. Acad. Sci. U.S.A. 106, 5942-5947. doi: 10.1073/pnas.0811431106

Sanders, J., Cowansage, K., Baumgartel, K., and Mayford, M. (2012). Elimination of dendritic spines with long-term memory is specific to active circuits. J. Neurosci. 32, 12570-12578. doi: 10.1523/JNEUROSCI.1131-12.2012

Shepherd, J. D., and Bear, M. F. (2011). New views of Arc, a master regulator of synaptic plasticity. Nat. Neurosci. 14, 279-284. doi: 10.1038/nn.2708

Shepherd, J. D., Rumbaugh, G., Wu, J., Chowdhury, S., Plath, N., Kuhl, D., et al. (2006). Arc/Arg3.1 mediates homeostatic synaptic scaling of AMPA receptors. Neuron 52, 475-484. doi: 10.1016/j.neuron.2006.08.034

Steward, O., Wallace, C. S., Lyford, G. L., and Worley, P. F. (1998). Synaptic activation causes the mRNA for the IEG Arc to localize selectively near activated postsynaptic sites on dendrites. Neuron 21, 741-751. doi: 10.1016/S08966273(00)80591-7

Tanaka, K. Z., Pevzner, A., Hamidi, A. B., Nakazawa, Y., Graham, J., and Wiltgen, B. J. (2014). Cortical representations are reinstated by the hippocampus during memory retrieval. Neuron 84, 347-354. doi: 10.1016/j.neuron.2014.09.037

Thompson, C. L., Wisor, J. P., Lee, C. K., Pathak, S. D., Gerashchenko, D., Smith, K. A., et al. (2010). Molecular and anatomical signatures of sleep deprivation in the mouse brain. Front. Neurosci. 4:165. doi: 10.3389/fnins.2010.00165

Tse, D., Takeuchi, T., Kakeyama, M., Kajii, Y., Okuno, H., Tohyama, C., et al. (2011). Schema-dependent gene activation and memory encoding in neocortex. Science 333, 891-895. doi: 10.1126/science.1205274

Turrigiano, G. G. (2008). The self-tuning neuron: synaptic scaling of excitatory synapses. Cell 135, 422-435. doi: 10.1016/j.cell.2008.10.008

Urban, D. J., and Roth, B. L. (2015). DREADDs (designer receptors exclusively activated by designer drugs): chemogenetic tools with therapeutic utility. Annu. Rev. Pharmacol. Toxicol. 55, 399-417. doi: 10.1146/annurev-pharmtox-010814124803 
Vann, S. D., Brown, M. W., Erichsen, J. T., and Aggleton, J. P. (2000). Fos imaging reveals differential patterns of hippocampal and parahippocampal subfield activation in rats in response to different spatial memory tests. J. Neurosci. 20, 2711-2718.

Vazdarjanova, A., and Guzowski, J. F. (2004). Differences in hippocampal neuronal population responses to modifications of an environmental context: evidence for distinct, yet complementary, functions of CA3 and CA1 ensembles. J. Neurosci. 24, 6489-6496. doi: 10.1523/JNEUROSCI.0350-04.2004

Vazdarjanova, A., McNaughton, B. L., Barnes, C. A., Worley, P. F., and Guzowski, J. F. (2002). Experience-dependent coincident expression of the effector immediate-early genes arc and Homer 1a in hippocampal and neocortical neuronal networks. J. Neurosci. 22, 10067-10071.

Wallace, C. S., Lyford, G. L., Worley, P. F., and Steward, O. (1998). Differential intracellular sorting of immediate early gene mRNAs depends on signals in the mRNA sequence. J. Neurosci. 18, 26-35.

Wang, K. H., Majewska, A., Schummers, J., Farley, B., Hu, C., Sur, M., et al. (2006). In vivo two-photon imaging reveals a role of arc in enhancing orientation specificity in visual cortex. Cell 126, 389-402. doi: 10.1016/j.cell.2006.06.038

Wang, S. H., Redondo, R. L., and Morris, R. G. (2010). Relevance of synaptic tagging and capture to the persistence of long-term potentiation and everyday spatial memory. Proc. Natl. Acad. Sci. U.S.A. 107, 19537-19542. doi: 10.1073/pnas.1008638107

West, A. E., Chen, W. G., Dalva, M. B., Dolmetsch, R. E., Kornhauser, J. M., Shaywitz, A. J., et al. (2001). Calcium regulation of neuronal gene expression. Proc. Natl. Acad. Sci. U.S.A. 98, 11024-11031. doi: 10.1073/pnas.191352298

West, A. E., and Greenberg, M. E. (2011). Neuronal activity-regulated gene transcription in synapse development and cognitive function. Cold Spring Harb. Perspect. Biol. 3:a005744. doi: 10.1101/cshperspect.a005744
Whitlock, J. R., Heynen, A. J., Shuler, M. G., and Bear, M. F. (2006). Learning induces long-term potentiation in the hippocampus. Science 313, 1093-1097. doi: $10.1126 /$ science. 1128134

Wiltgen, B. J., Zhou, M., Cai, Y., Balaji, J., Karlsson, M. G., Parivash, S. N., et al. (2010). The hippocampus plays a selective role in the retrieval of detailed contextual memories. Curr. Biol. 20, 1336-1344. doi: 10.1016/j.cub.2010. 06.068

Yamada, K., Homma, C., Tanemura, K., Ikeda, T., Itohara, S., and Nagaoka, Y. (2011). Analyses of fear memory in Arc/Arg3.1-deficient mice: intact shortterm memory and impaired long-term and remote memory. World J. Neurosci. 1, 1-8. doi: 10.4236/wjns.2011.11001

Yassin, L., Benedetti, B. L., Jouhanneau, J. S., Wen, J. A., Poulet, J. F., and Barth, A. L. (2010). An embedded subnetwork of highly active neurons in the neocortex. Neuron 68, 1043-1050. doi: 10.1016/j.neuron.2010.11.029

Zhang, W., Wu, J., Ward, M. D., Yang, S., Chuang, Y. A., Xiao, M., et al. (2015). Structural basis of arc binding to synaptic proteins: implications for cognitive disease. Neuron 86, 490-500. doi: 10.1016/j.neuron.2015.03.030

Conflict of Interest Statement: The authors declare that the research was conducted in the absence of any commercial or financial relationships that could be construed as a potential conflict of interest.

Copyright (C) 2016 Minatohara, Akiyoshi and Okuno. This is an open-access article distributed under the terms of the Creative Commons Attribution License (CC BY). The use, distribution or reproduction in other forums is permitted, provided the original author(s) or licensor are credited and that the original publication in this journal is cited, in accordance with accepted academic practice. No use, distribution or reproduction is permitted which does not comply with these terms. 